\title{
Chronic Pain and Depression in Low Back (Spinal) Injured Patients
}

\author{
Vito Zepinic $^{1}$, Blagoj Kuzmanovski ${ }^{2}$ \\ ${ }^{1}$ PsychClinic $\mathrm{P} / \mathrm{L}$, London, United Kingdom \\ ${ }^{2}$ Family Medical Clinic, Sydney, Australia
}

Email address:

vito@psychclinic.net (V. Zepinic)

\section{To cite this article:}

Vito Zepinic, Blagoj Kuzmanovski. Chronic Pain and Depression in Low Back (Spinal) Injured Patients. American Journal of Applied Psychology. Vol. 8, No. 5, 2019, pp. 89-97. doi: 10.11648/j.ajap.20190805.11

Received: August 22, 2019; Accepted: September 11, 2019; Published: September 24, 2019

\begin{abstract}
Depression caused by physical dysfunction and associated symptoms as the aftermath of the low back (spinal) injury is commonly undiagnosed and untreated. In this paper, based on our clinical experience, we have described a relation between depression and lumbosacral injury. In our research, we selected 54 ( 26 female and 28 male) patients in two different groups: (1) those whose low back injury occurred less than 12 months before research commenced; and (2) those whose injuries occurred more than 12 months before the research (their condition has been considered as a chronic). All of the patients ( $\mathrm{n}=54)$ were assessed by using the Beck Depression Inventory (BDI), Montgomery-Asberg Depression Rating Scale (MADRS), and General Health Questionnaire-28 (GHQ-28) in order to evaluate their level of depression. The results show that low back (spinal) injured patients suffered depression due to their body discomfort and changed day-to-day capacities. Those who sustained injury more than one year developed an upper level of a moderate depression. However, if not taking any treatment following their psychological disturbances, the patients had suffered a severe depression. Their level of depression increases with a chronicity of the physical pain making also depression as a chronic disorder.
\end{abstract}

Keywords: Depression, Low Back Injury, Psychosomatic Symptoms, Dysfunction

\section{Introduction}

Depression and related psychosomatic dysfunctions in patients with the low back (spinal) injury is quite commonly undiagnosed and untreated despite causing severe, significant dysfunctions in day-to-day functioning, and negative effect on patient's health. Despite its treatability, depression which is an aftermath of physical injuries is frequently undiagnosed for several reasons. One of the most common is that many symptoms of the depression are difficult to determine whether such symptoms are manifestations of the physical injury or they are a comorbid depressive disorder.

Furthermore, as the physical injuries commonly caused discomfort, it can be difficult to differentiate normal, adaptive psychological reactions from dysfunctional psychological states and overt psychiatric illness [1]. From diagnostic point of view, it is difficult to distinguish extent of the patient's passive suicidal ideation caused by chronic pain and to what extent it reflects the patient's wish to escape from such unbearable suffering or from a rational attempt to end his dysfunctional life. In general, it is clear that depressive disorders occur increasingly in chronic low back pain, have an adverse effect on quality of the patient's life, and increased patient's morbidity. Another issue that emerges from psychological theories of pain is the importance of emotional responsibility responses and pain beliefs. Patients with low back injury show tremendous differences in their ability to regulate emotions as well as their attributions about pain, their judgments about the seriousness of pain, their expectations of assistance and emotional support from others, and their sense of control and mastery over pain [2].

Pain and physical distress, limitations in daily functioning and incapacity to work, and to engage in pleasurable activities, perceived alteration in the anticipated life expectancy, fears of disability and dependency, alteration in intimate relationship, family and social life, inevitable bring the sufferer in experiencing sadness, despair, lower mood, helplessness and hopelessness [3]. DSM-5 [4] had recognised depressive disorder caused by the medical condition with three main elements of the diagnostic criteria: (1) prominent 
and persisted period of depressed mood or markedly diminished interest or pleasure in all or, almost all, activities that predominates in the clinical picture; $(2)$ there is evidence from the history, physical examination, or laboratory findings that the patient's disturbance is the direct pathophysiological consequences of another medical condition; and (3) the disturbance causes clinically significant distress and/or impairment in social, occupational and/or other important areas of functioning.

\section{Psychosomatic Symptoms of Spinal Injury}

Low back pain is one of the most common causes of neurological and neurosurgical consultations. We define lower back pain as the pain caused by injury on lumbosacral spine (areas of L1-5 and S1-5) as being either a radiculopathy, or a mechanical low back pain. In medical practice low back pain is closely related to low limb pain. Diagnosis is suspected in patients with the low back pain that is exacerbated by standing and walking and relieved promptly by sitting, and pain radiates down in a distribution appropriate to the involved nerve root [5]. Low back pain is multifactorial and most commonly caused by a peripheral nerve root compression from the intervertebral disc protrusion, bulge, or an intraspinal tumour. Compression may be within the spinal canal or intervertebral foramen by disc protrusion, tumour, or bony irregularities (e.g., osteoarthritis). Psychiatric factors must be evaluated as a central or associated issue early in the illness [6].

In medical populations, depressive symptoms associated to physical injury may manifest in atypical or marked forms, including the amplification and noncompliance with, or refusal of needed medical treatment. Physical suffering, such as a low back (spinal) injury and disability, diminishes patient's capacity to experience pleasure in many activities, what are usually characteristic symptoms of depression. It is common that, not until pain and other physical symptoms have been adequately treated which could improve patient's physical capacity, the depression is diagnosed as an aftermath of the chronic pain condition. A depressed mood or withdrawal from social activities that is disproportionate to physical disability increase the likelihood that depressive disorder is present [3].

Depression in the spinal injured patients is frequently undiagnosed and untreated despite evident impact upon the patient's mental health. It is clear that apathy, fatigue, sadness, sleep disturbances, and an adverse effect on life are common outcomes. The experience of sadness is a normal, expectable response to the spinal cord injury due to the changes in bodily appearance, and functioning. Chronic low back pain is usually defined as a complex state in which the pain symptoms have persisted long after the original spinal injury or trauma onset (often described as longer than six months). The symptoms are viewed as exceeding the typical expected time course for healing of the acute injury, and the symptom intensity and duration are also viewed as in excess of what would be predicted by the medical condition alone. Patients with low back pain suffer dramatic reductions in their physical, social, and psychological well-being with lowered health-related quality of life [3].

Some researchers have noted that the patient's pain symptoms move beyond acute injury to a point where the pain symptoms themselves become the disease. Chronic low back pain is usually associated with a broad array of the functional impairments, psychological symptoms, disability, and a high rate of medical service utilisation. Patients with this type of chronic pain are often referred for psychological or psychiatric services and compose significant subgroup. Psychological factors such as depressed mood, distress, and somatisation are highly correlated with low back pain, which predict the transition from acute into the chronic pain condition, and chronic condition of depression [7].

Patients with chronic low back pain exemplify the complexity of treatment, which should be holotropic and multidisciplinary. However, it is common that the multidisciplinary treatment plan for the chronic low back pain is not implemented, and it is based only on unidimensional biomedical models with emphasis on the correct diagnosis and treatment using procedures such as the nerve blocks, massage, pharmacological approaches, and/or surgery, without consideration of coexisting psychosocial factors. Furthermore, physical symptoms alone are often poorly associated with a predictive value in return to work or functional outcomes. There is no doubt that severe low back injury is a stressful experience which impacts as a psychological condition.

Stressful events, such as severe physical injury and disability, have subjective meanings in forms of loss and fear, which are important in the aetiology of depression, anxiety, specific phobias, substance abuse, and personality disorders. Psychological trauma with physical injuries is also largely focused on development of Posttraumatic Stress Disorder (PTSD). However, the other psychiatric outcomes are less investigated; specifically, the trauma experienced by subjects who attended the emergency department as victims of a motor vehicle or work-related accidents. As outcomes they have shown immediate onset of the variety of psychiatric complications and disorders - general anxiety, depression, cognitive impairment, specific phobias, and other stressrelated disorders. These issues could even not be fully recognised by the law in compensation claims due to a fear of the "floodgate of liability claims" [8].

Symptoms of depression associated with a chronic low back pain are generally defined to include depressed mood, crying spells, depleted self-esteem, lack of the selfconfidence, thoughts of death and suicide, disturbances in sleep (insomnia or hypersomnia), changes in appetite (anorexia or bulimia), disturbances in energy and activity levels, anhedonia (inability to experience pleasure), loss of concentration, the short-term memory problems, sexual dysfunctions, and social withdrawal [3]. Such symptoms are frequently seen in chronic low back pain patients and lead to 
question about the incidence of clinical depression in the chronic pain. In a review of rates of depression in chronic pain there is reported prevalence range from $10 \%$ to $100 \%$ across the studies, which likely reflects methodological problems in diagnostic and assessment techniques in assessing the impact on a mental health of injured people.

Various approaches which proposed to diminish effect of the confounding medical symptoms in the diagnosis of depression - the DSM-5 [4] - suggest an "exclusive" and "aetiological" approach, which specifies that symptoms are judged by the clinicians to be aetiologically related to a general condition and then symptoms should be excluded from the diagnostic criteria of a major depressive disorder. However, this approach does not specify whether the clinicians should focus of the physiological state as consequences of medical condition (low back pain), or extend to psychological reactions of the condition.

Depression and other associated disorders caused by the low back injuries are often left undiagnosed and this is of concern because missing the diagnosis of depression, or a minor depressive disorder, may result in the lost opportunity to improve quality of life, the risk of suicide, shorten hospital stay, and improve treatment compliance. It is also of note that specialists seeing patients with low back injuries frequently are unaware of the patient's emotional distress and needs to evaluate that. Some clinicians may fear that they lack a sufficient time or skill to manage the emotionality that may be triggered when they inquire about patient's emotional reaction to the pain experience. Some patients may be reluctant to disclose depressive symptoms because of perceived stigma or perceived lack of interest by their primary medical caregivers [9]. Also, some patients may not even recognise depressive symptoms that are present, or may attribute such symptoms to the effects of their only medical (physical) condition.

There is considerable controversy in how to diagnose depression and/or other psychiatric disorders with patients who have sustained a chronic low back pain. The most common dilemmas are: (1) whether depressive symptoms are "appropriate" to stress and disability; (2) many symptoms of depression are similar to a medical illness itself; and (3) symptoms of depression often overlap with the other psychiatric disorders [3]. Over the past two decades, there have been numerous investigations of affective aspects of the low back pain experience and to see the role of emotional distress, catastrophic coping strategies, self-efficacy, and lack of motivational factors in persistent pain.

Clinical experience shows considerable overlap of PTSD symptoms and other psychiatric disorders with victims of the accident who, nevertheless, present significant distress and disabling. The relationship between stressful life events and risk of a major depression is largely presented. In this study, we used a measure to find the cause between the long-term disabling adversity and the depression. There is growing evidence that people with chronic low back pain avoid a variety of stimuli, including those directly (physical activities) or indirectly (social activities) due to their pain.
The low back pain is conceptualised as a complex, subjective, perceptual phenomenon that involves a number of dimensions such as intensity, quality, time course, and personal meaning. Pain of this nature usually leads to significant distress, suffering, and functional disability.

Using standardised diagnostic criteria, [10] in a study of the depression prevalence among men with the chronic low back pain, compared with a control group without back pain history, found significantly elevated depression $(32 \%$ versus $16 \%)$, alcohol abuse (65\% versus $39 \%)$, and major anxiety disorder (31\% versus $14 \%)$. Further, they also concluded that more major depressive episodes developed after the onset of pain than as one pre-existing condition. However, workrelated low back injuries pose different subjective psychological demands than other condition, such as are gradual-onset of pain disorders.

Severity and character of the low back pain, response to the pain-relief measures, patient's functional limitations in daily activities, and changes in marital, family, or employment role may all affect patient's self-perceptions and affects state. Individuals whose pain appraisal involves negative thought patterns and coping mechanisms have been found to increase rates of depression and anxiety.

Unrealistic expectations and unawareness of a mental health condition caused by the low back pain play a major role in exaggerated fears, and avoidance. Avoidance refers to a pattern of behaviour that delays, or puts off (ignores), undesirable situation or experience. Avoidance is an important factor in the maintenance of disability in patients with chronic low back pain. Although avoidance may be adaptive in response to chronic pain, its long-term effects are deleterious.

Social avoidance behaviour due to discomfort caused by chronic low back pain is related to accurate expectations (as in pain-phobic patients), that an activity is more painful than it is actually the case. This in turn is related to fearing and avoidance of the particular day-to-day activities, such as climbing few stairs or using support stick to assist in walking. Furthermore, due to the avoidance as a secondary outcome, the patients suffered from muscle tightening and wasting, support patient's further reinforcement for avoidance of any physical activities. Fear-avoidance of the physical activities usually leads to the self-isolation and social withdrawal. Pain-stricken patient who behaves anxiously in the context of his chronic pain entails a number of unhelpful responses such as reduced range of the emotions, and decreasing coping with the pain.

During clinical interview with the low back patients, questions that may be helpful for generating a diagnosis of depression would be: What are you able to enjoy these days? What interests you now? When did you last have a good time? Patients who report a loss of the interest and pleasure for at least two weeks should be considered as those who are suffering symptoms of depression, and they are likely candidates for the diagnosis of the depression, or depressed mood. It is also helpful to investigate the presence of other psychological symptoms of depression such as an inability to 
concentrate, the sense of lowered self-esteem or feelings of guilt, and thoughts of the self-harm. As a part of mental status examination, it is important to evaluate the levels of short-term memory span, or memory deficits. Severity of the psychological symptoms should be judged based on the degree of distress and functional disability, and also to reassure absence of the psychotic features.

It is common that chronic low back pain and subsequent discomfort cause either a loss of pleasure in all, or most, activities or lack of reactivity to usual pleasurable stimuli. Patient's melancholic features are accompanied by three or more of the following: (1) distinct quality of depressed mood different from bereavement; (2) depression is regularly worse in the morning; (3) insomnia with early morning awakening; (4) hypoactive sexual desire; (5) marked psychomotor retardation or agitation; (6) interpersonal rejection sensitivity;

(7) significant changes in appetite [4].

In this study, we tried to find a relationship between chronic low back pain and depression using objective psychometric testing and clinical interview. Three major factors were targeted to evaluate the pain-depression relationship:

i. perceived interference (adverse effect of pain and activities);

ii. measures of the cognitive distortions (negative selfstatement, depleted self-evaluation, catastrophic, overgeneralisation); and

iii.perceived level of the self-control (perceptions of control and adjustment versus hopelessness).

The chronic pain-depression relationship demands characteristics of living with chronic pain that produces patient's negative cognitive, and personality changes, which often leads to depression. Chronic low back pain is itself stressor which can lead to sufficient changes causing symptoms of major depression or, if depressive symptoms persist more than two years, to the dysthymic disorder. We predict that measuring depressive symptoms would prove more information about the pain and disability than would relay on measures of single pain presentation. The depressed patients' negative outlook is often a source of their frustration to friends, family, and clinician when they try to be of help. Chronical depression makes patients' view for the future as black and hopeless, and believe that none of their problems can be solved. In severe cases, suicidal wishes tend to be intense; they feel hopeless and helpless, and "there is only one way out - to kill myself".

\section{Method}

\subsection{Participants}

Subjects to this study were 54 patients with low back injury referred to our Clinic following their psychological disturbances (depression, anxiety) caused by their pain. About half of the patients (44\%) were female. Most of the referred patients were married $(85 \%)$ and middle-aged $(\mathrm{M}=41.3 ; \mathrm{SD}=12.4)$, and most of them $(89 \%)$ had completed the secondary education or professional course.

Referred patients had mostly complained of the low back pain $(76 \%)$ followed by the lower extremity (legs) pain $(19 \%)$, and pain on the other body location (4\%). The median reported duration of the pain was nine months (range 1-27 months). Subject were divided into two groups: 23 who sustained injury on low back pain between 6-12 months prior to the referral to our Clinic, and 31 those who had an injury more than 12 months before referral was made.

All referred patients had previously been diagnosed having thoraco-lumbar spine injury (disc bulge, herniation, protrusion, or disruption) confirmed by CT scan or/and MRI test. Before proceeding with the examination, from the all patients an informed consent was obtained using data of the psychometric testing for research, but not releasing identity of anyone.

\subsection{Measures}

All referred patients were screened (although some of them already being diagnosed with depression and had been prescribed medication) with the following instruments:

i. Beck Depression Inventory (BDI);

ii. Montgomery-Asberg Depression Rating Scale (MADRS); and

iii.General Health Questionnaire-28 (GHQ-28)

Beck Depression Inventory (BDI) has been developed to measure the behavioural manifestation of depression in both adults and adolescents [11]. It is designed to standardise the assessment of depression severity in order to monitor changes over time, either with or without therapy provided, or to simply describe the illness. The items of the BDI were originally derived from clinical observations of depressed patients made during the course of psychoanalytic psychotherapy. Attitudes and symptoms that appeared to be specific to the depressed patients were described by a series of the statements, and a numerical value was assigned to each statement.

In its original form, BDI covers 21 behavioural manifestations, each area represented by four $(0-3)$ statements describing symptoms severity from a low to a high. Patients were asked to identify the statement that best describes their feelings "right now". Items were then scored and summed to obtain a total score for the severity of depressed symptoms. The most recent guideline suggests the following interpretation of severity scores: 0-9, minimal; 10 16, mild; 17-29, moderate; and 20-63, severe. Subscale scores may be calculated for a cognitive-affective factor and a somatic factor.

The BDI is self-questionnaire which takes patient's 5-10 minutes to answer on all items, however in some severely depressed or obsessed patients, administration time may be somewhat longer. Correlations between the BDI and other standard measures of the depressive symptom severity show high, but not complete, concordance across measures. Correlations between clinical ratings of depression and the BDI for psychiatric patients range from 0.55 to 0.96 , with a mean of 0.72 . For non-psychiatric subjects, correlations 
range from 0.55 to 0.73 , with a mean of 0.60 .

Beck [12] described that the symptomatology of depression is not a simple entity but combination of the emotional (dejected mood, self-dislike, loss of gratification, loss of attachment, crying spells, loss of mirth response), cognitive (low self-evaluation, negative expectations, selfblame and self-criticism, indecisiveness, distorted self-image, loss of motivation, suicidal wishes or attempts, hopelessness, loss of interest, dependency), and vegetative and physical manifestations (fatiguability, sleep disturbances, loss of appetite, loss of libido).

Montgomery-Asberg Depression Rating Scale (MADRS) was designed to be used in patients with major depressive disorder to measure an overall severity of depressive symptoms [13]. While developing scale, the authors have two goals: (1) to design a scale that would assess treatmentsensitive change, and (2) to construct an instrument that could be used by both psychiatrists and professionals without specific psychiatric training.

The MADRS is designed from the Comprehensive Psychopathological Rating Scale with selected items associated with major depressive disorder. It is a 10 -item checklist and its items are related on a scale of 0-6 with anchors at 2-points interval. It takes approximately 15 minutes to administer and the MADRS copy rights belong to the British Journal of Psychiatry which first published the scale. Alongside with medically training assessors, the scale can be used by those clinicians with a minimal training and those who are not mental health professionals. It was found [14] that the symptoms assessed by the MADRS in 70\% were present in patients who met the Research Diagnostic Criteria for a major depressive disorder.

The MADRS is useful scale of the degree of symptoms severity in depressed patients. Its particular utility is in evaluating changes in symptom severity, such as those occurring with treatment. Because of its reliance on cognitive features of major depression, it is less likely to misdiagnose depression in patients with general medical condition.

General Health Questionnaire-28 (GHQ-28) was designed to assess the presence of psychiatric distress related to general medical illness. This questionnaire is not designed for the psychiatric diagnosis but rather as a screening instrument that measures condition which should be formally evaluated to determine psychiatric diagnosis. The GHQ-28 may be seen as a complement to the formal psychiatric interview typically conducted by the clinicians; it is possible to state the number of symptoms at which there is a $95 \%$ probability that an individual will meet the criteria for a psychiatric case [15].

The authors of the GHQ-28 used a one-dimensional model of psychiatric "caseness" rather than an official diagnosis to explain patient's scores on the GHQ-28. The questionnaire was developed to evaluate the psychological components of ill health. It assesses a patient's ability to carry out his daily functions and the emergency of a new psychological disorder rather than lifelong personality characteristics.

Alongside with assessment using the above-stated questionnaires, we also used clinical interview to assess the severity of symptoms, changes over time, and the efficacy of prescribed medication, taking into account the patient's clinical condition and the severity of side effect (particularly the long use of painkillers). It was designed to assist the clinician an impression of the patient's current illness state, and assessing patient's improvement or worsening over time. In general, questions during the interview were almost the same addressed to all patients, in particular the statement about how the current condition interferes with patient's functioning. During the interview time, it was also important to observe patient's body language: the presence of posturing, grimacing while moving or crossing legs, or other gestures that occurred.

The presence of depression in a patient with chronic low back pain is associated with decreased function, poorer treatment response, and increased health care costs [16]. For example, in patient with severe low back pain, a cycle of the excessive fear ("pain catastrophising") of movement leading to deconditioning, further worsening pain and further fear termed fear avoidance - has been often found and more predictive of disability than real pain intensity. In primary care, the physicians often focus more on the sensory aspects of the pain (e.g. intensity) but less, or even not at all, on the emotional distress as the most disruptive features. However, some clinicians [17] found that average of $52 \%$ of patients with pain fulfil the criteria for depression (negative mood, hopelessness, despair, etc.).

We focused our clinical interview to evaluate symptoms that fulfil the DSM-5 diagnostic criteria A-C for depression [4]. The criterion was that symptoms are present nearly every day, with the exception of weight changes and suicidal ideation (or attempt). However, if patient reported frequent or recent suicidal ideation, the interviewer then would evaluate patient's condition in depth and prioritise treatment to this problem.

In clinical practice, it is common that patients deny depression as a comorbid condition of low back pain ant that their essential features (depressed mood, insomnia or hypersomnia, sadness, hopelessness, diminished interest or pleasure in activities, loss of energy or fatigue, psychomotor agitation) are due to a physical discomfort not an emotional distress. Many patients will report or exhibit increased irritability due to their "physical discomfort" (e.g., persistent anger, tendency to respond to the events with angry outbursts or blaming others, exaggerated sense of frustration over minor matters) although these symptoms are typical for depression not for their low back pain. Loss of interest or pleasure is nearly always present, or not feeling any enjoyment in activities that were previously considered pleasurable, but patients will blame physical discomfort (pain) for their inability to be active.

\section{Results}

Despite some criticisms that the measuring depression using BDI in people with physical disabilities can be 
problematic because of items about work, tiredness, and weight loss or gain, the BDI has been reported to be a valid and sensitive instrument. We used four-points recommended scale to measure results in assessing the severity of depression with low back pain patients for both questionnaires (BDI and MADRS) between two groups of the patients. We hypothesised that an individual's response to the chronic pain reflects characteristics of the pain and the person's thoughts and behaviour developed during the course of the illness, which are subject to both positive and negative reinforcement. The patient's challenges of chronic pain, which are commonly described, include decreased enjoyment of normal activities, loss of function, role change, and relationship difficulties.

The scores of psychometric testing regarding patients' level of depression is evident in the Table 1 below.

Table 1. Depression level assessed by BDI and MADRS.

\begin{tabular}{lll}
\hline & BDI & MADRS \\
\hline$<12$ months of injury & 17.8 & 21.4 \\
$>12$ months of injury & 25.3 & 31.4 \\
\hline
\end{tabular}

We found that both groups of the patients who suffered lumbosacral injury reported symptoms of depression. However, patients who had the injury for a period of less than 12 months before the assessment were categorised as average having "mild-to-moderate" level of depression. On the other hand, those patients who had the injury for more than 12 months before the assessment, placed at upper level of a "moderate" level of depression.

When moderately depressed patients were divided into two subgroups (those who had received treatment regarding psychological condition (depression, anxiety) and those who did not have any treatment prior being referred to our Clinic), we found interesting and significant differences (Table 2).

Table 2. Differences in depression between treated and not treated patients.

\begin{tabular}{lll}
\hline & BDI & MADRS \\
\hline With treatment $(\mathrm{N}=19)$ & 21.2 & 20.8 \\
Without treatment $(\mathrm{N}=35)$ & 36.5 & 42.4 \\
\hline
\end{tabular}

Above results revealed that patients who did not have any treatment regarding their depressed state scored results which placed them in the category of severe depression. The results also confirm that despite having treatment, patients who had the injury for more than 12 months prior to the assessment, presented a higher level of depressive symptoms than those who had the injury less than 12 months before the assessment.

From the research and clinical practice, it is evident that depression is highly correlated with the low back pain. Specifically, anxiety, depression, and occupational mental stress predicted lower rates of return to work in patients with lumbosacral injuries. In a 23-year follow-up study [18] that examined the longitudinal relationship between low back pain and depressive disorder, using lifetime reports of symptoms, and excluded other forms of affective distress such as demoralisation, grief, and adjustment disorder, it was found that depressive disorder was a significant risk factor for incident low back pain. The level of depression increases with a chronicity of the pain making also depression as a chronic disorder. In case stated below is typically presented patient with both chronic pain and chronic depression as an aftermath:

Ms C, a 34-year-old nurse, was refereed to our Clinic for a treatment due to her severe depression as an aftermath of low back injury. She suffered severe injury after she slipped in her bathroom and, as a result, she needed operation on her lumbar spine but the low back pain has continued. During interview and psychometric testing, she presented with severe cognitive and emotional difficulties with very poor coping mechanisms to deal with her physical disability and depression. Psychometric tests revealed chronic impairment in emotionality, judgment, impulse control, interpersonal relationships, sleeping problems, and significantly decreased sexual desire. Her interpersonal boundaries were weak, with marked evidence of loss of the self-confidence. Her relationships were typically short-lived, tense, and quarrelsome.

Ms C reported many inconsistent and incompatible symptoms and could led to endorse self-blame and nonphysiological complaints. Experience of severe sadness, expectable response to the multiple adverse effects of a serious mood changes were also evident. The effects include changes in bodily appearance and functioning; pain and physical distress; limitations in the capacity to work and to engage in pleasurable activities; fears of disability; alterations in intimate relationships, family life, social relationships, and activities.

Using clinical interview and psychometric testing (BDI and MADRS) we have summarised depressive symptoms in low back pain patients into four categories that should be treated:

i. symptoms of the medical illness (e.g., fatigue, weight loss or gain, fatigue, apathy, sleeping problems, psychomotor retardation) that cause a variety of the emotional disturbances ("emotionalism");

ii. suicidal thoughts or self-harm that are linked to hopelessness and unrelieved pain;

iii.diminished capacity or loss of interest to experience pleasure in many activities; withdrawal from social or physical activities that is disproportionate to the physical disability; and

iv.manifestation of the atypical or masked forms, including amplification of somatic symptoms and noncompliance with or refusal of medical treatment (these factors may contribute to both the underdiagnosis and the overdiagnosis of depression).

In the evaluation, the GHQ-28 was used as complementary to the psychiatric interview to assess psychological components of the chronic low back pain. We divided the scores into four subscales (A-somatic symptoms, Banxiety/insomnia, C-social dysfunction, D-severe depression) to evaluate chronicity of the reported symptoms (Table 3). These results show differences between patients who had 
suffered low back injury less than one year and longer than one year, as well as the differences between those who had or had not have a treatment regarding their injury.

Table 3. GHO-28 results between treated and not treated patients.

\begin{tabular}{llll}
\hline Scale & $<$ 1 year of injury & $\begin{array}{l}\text { >1 year of injury } \\
\text { with therapy }\end{array}$ & $\begin{array}{l}>1 \text { year of injury } \\
\text { with no therapy }\end{array}$ \\
\hline Scale A & 14.2 & 24.6 & 21.8 \\
Scale B & 13.5 & 18.1 & 22.1 \\
Scale C & 15.8 & 25.2 & 24.5 \\
Scale D & 15.2 & 24.3 & 25.8 \\
\hline
\end{tabular}

We found interesting differences among three groups: patients who had the injury less than 12 months before the examination scored the "consistent" results on all four scales. However, patients who had the injury more than 12 months before the examination and had received therapy due to their psychological condition, showed "inconsistent" scores. They have higher scores on Scale A (somatic symptoms) and Scale $\mathrm{C}$ (social dysfunction) than the patients who did not have any treatment.

These results revealed the patients' disappointment with therapy provided and that no progress has been achieved, confirming the chronicity of their pain and accompanied problems (depressive symptoms). On further evaluation, they reported that their physical condition and depression are much worse than after the injury; they feel run down, more incapable of making decision, cannot enjoy their normal dayto-day activities, and they are unsatisfied with the progress of work carried out regarding their condition.

High score on Scale D (severe depression) confirmed severity of psychological distress caused by a chronic pain condition associated with the patients' feelings that life is not worth living; they feel worthless, hopeless, and that things are not on their side. Severe depression often violates the autonomy of the patient at the level of body integrity. The injured body is seen worthless and dysfunctional which may destroy self-confidence and self-continuity. At the same time, depression may affect patients by causing them both to withdraw from close relationships and disruption of basic trust.

Low back injury accompanied with severe depression is often associated with decreased sexual desire or sexual dysfunction. Treatment for that problem involves much more than the medical management of the condition. It requires extensive psychological adjustment to a new body image and altered self-concept. Frequently, the patients perceive own self as disfigured, helpless and hopeless. They may feel useless and burdensome to those around them. Socially, they feel isolated from others - they isolated themselves from others - and feel alone with their suffering and emotional pain. They are overwhelmed with the uncertainty of their future, for life as they once knew it has been a chronic physical but also psychological disturbances.

Chronic lumbosacral injury is a cardinal manifestation of the spinal injury, and the relief of pain is probably the most common demand made by the patient upon presentation to a physician. Patients who experience chronic low back pain often have a host of related symptoms. Some of these individuals are chronically depressed, pessimistic and gloomy, whose guilty and self-depreciating attitudes are readily apparent to the moment they walk into the clinician's office. They seem to have no joy or enthusiasm for life and, indeed, some seem to have suffered the most extraordinary number and variety of defeats, humiliations, and unpleasant experiences.

\section{Conclusions and Further Directions}

Depression is common in patients with chronic pain such as a low back injury. Making diagnosis can be difficult and is best done as part of a wider multidimensional pain assessment. Depression in low back patients is usually treated only pharmacologically, however the treatment efficacy is often reduced in patients with severe and prolonged pain (chronic condition). Collaboration with other treating clinicians and specialists are often useful, especially in complex cases to manage their day-to-day activities, and to improve function and quality of life.

The emotional response to the low back injury is very complex, highly individualised, and influenced by a number of intervening variables. Reactive depression is most frequently noted especially among individuals who were extremely active prior to the injury and relied upon physical activity to deal with problems in the past. The patient, in reactions to the injury, attempts to mobilise previous ways of coping, struggling to survive with this sudden and catastrophic trauma. Rather than a stable state, adjustment, for most patients, is a process that evolves over time; it is an individual response to the crisis as well as a reaction to loss of a variety of the normal functioning. During the process of adjustment, patient needs to reformulate a new strategy, and a new set of expectations and goals that must be substituted for the past gratification (see: Table 3). This is often an emotionally painful process that involves the grieving of losses and refocusing on one's expectations of the future [19].

In many cases with low back injury, the patient's emotional reaction may fluctuate from intense feelings of depression and anger. Patient may experience feelings of self-blame for the injury or may feel that others have disappointed them and abandoned them in their hope to recover. For the patients to manage their secondary conditions, it is necessary to provide education as to their aetiology and to teach adaptive techniques as even the most cooperative patients may become overly demanding in their attitude. Patients learn that they must still assume responsibility and meet certain expectations despite the severity of their injury. Numerous researchers have shown that physical symptoms improvement is correlated with the improvement of the depression symptoms, which suggest that the patient's ability to achieve depression remission may be directly related to the reduction of painful physical symptoms.

It must be added that negative self-concept and poor 
family relationships which existed before the injury generally persist after the injury and may even be exacerbated. But maintaining normal relationships, when they exist, is important and must be encouraged. Dysfunctional relationships, or underlying family psychopathology, and/or poor family systems should be addressed through the therapy when problems either predate the injury or surface after the rehabilitation program. However, the involvement of positive and supportive family member ("co-therapist" role) can improve patient's co-operation and further goals of the therapy [20].

Levels of depression in the examined subjects have been found to be significantly higher in those patients who suffer low back injury for 12 or more months before evaluation of their psychological condition. Study confirmed significantly increased levels of depression, hopelessness, and depleted self-esteem in patients after one year of injury despite even having medical treatment (see: Table 2). This finding confirms that the psychological adjustment to chronic low back injury does not improve within time and that psychological difficulties remain years after the injury [3].

The clinical interview we used, has been designed to identify how and in what degree the low back injury and chronic pain caused psychological distress and depressive condition. The score results on BDI and MADRS (see: Table 1) confirmed that they suffered from an accumulative stress, as injury caused their dysfunction in day-to-day activities, marital problems, sexual difficulties, patients' changes in social life and living condition, premature retirement, business readjustment, changes in number of arguments with a partner or separation, and changes in sleeping habits (insomnia or hypersomnia).

The severity and complexity of the low back pain show that it is not a uniform entity in its aetiology, or treatment. Results in this study confirm that, with low back pain, the patients' depression symptoms should be examined in anyway. It is important to discuss the details of the patient's complaints at the beginning of the clinical interview in order to reassure the patient of the clinician's belief about the stated complaints.

Obtaining subjective estimates of the patient's walking, sitting, and standing tolerance allows a comparison to be made with the imposed limits and the subjective estimates. Therefore, psychological data, whatever collected by clinical interview or psychometric testing, or both, should help to render statements referring to all five axes included in DSM-5 multiaxial classification [4]. Just as the depression is a frequent and somewhat natural reaction to a chronic pain, the effects of depression may make it more difficult to recover from pain.

The general principles that may be summarised from our research are as follows:

i. chronic low back pain is also a psychological phenomenon, which gives rise to identifiable qualities that permit its perception;

ii. chronic low back pain plays a significant role in the development of depressive symptoms and accompanied problems (dysfunctions); and iii.the severity of depressive symptoms is related to time since the onset of chronic low back pain, and may not be initially evident.

Although more interview time is required in primary care while attempting to evaluate the psychological condition of patients with the chronic low back pain, we cannot rule out this problem. A proper evaluation of the patient's physical condition should be structured to permit the patients to spontaneously reveal personal and psychological struggling along with the physical symptoms, and this gives more information for the appropriate multi-dimensional approach in the treatment modalities/options [21]. Recognising that a patient with low back injury is depressed, frustrated by persisting pain or having severely limit movements and activity are reasons to adopt more psychological or multidimensional approach than being only treated pharmacologically [22]. In their research [2] were of opinion that effective strategies for coping with persistent, recurrent, or chronic pain are very different from those for managing acute pain, and pain that persists beyond a few weeks can led to emotional and behavioural consequences that are deleterious to pain recovery and functional rehabilitation.

\section{References}

[1] Rodin, MG. Nolan, RP. Katz, MR.: Depression, In Levenson, JA. (ed.): Textbook of Psychosomatic Medicine, American Psychiatric Publishing, Washington DC, 2005.

[2] Linton, SJ. \& Shaw, WS.: Impact of Psychological Factors in the Experience of Pain, Physical Therapy, 5, 2011.

[3] Zepinic, V.: Post-injury chronic low back pain and depression: Comparative study between early and late post-injury sufferers show significant differences, International Journal of Health Science, 3, 2009.

[4] American Psychiatric Association: Diagnostic and Statistical Manual of Mental Disorders (DSM-5), APA, Washington DC, 2013.

[5] Bradley, WG. Daroff, RB. Fenichel, CM. Jankovic, J. (eds.): Neurology in Clinical Practice, $4^{\text {th }}$ ed, Butterworth \& Heinemann, Philadelphia, 2004.

[6] Berkov, R. \& Beers, MH. (eds.): The Merck Manual, $19^{\text {th }}$ ed, Merck \& Co, New York 1999.

[7] Pincus, T. Burton, AK. Vogel, S. Field, AP.: A systematic review of psychological factors as predictors of chronicity/disability in prospective cohort of low back pain, Spine, 25, 2002.

[8] Zepinic, V.: Fear of the "Floodgate of Liability" and Acknowledgment of the Recognisable Psychiatric Damage into English Law of Tort, Canadian Social Science, 4, 2015.

[9] Levenson, JA. (ed.): Textbook of Psychosomatic Medicine, American Psychiatric Publishing, Washington DC, 2005.

[10] Atkinson, JH. Slater, MA. Patterson, TL. Grant, I. Garfin, SR.: Prevalence, onset, and risk of psychiatric disorders in men with chronic low back pain, Pain Medicine, 45, 1991. 
[11] Beck, AT. \& Steer, RA.: Beck Depression Inventory Manual, Psychological Corporation, San Antonio, 1993.

[12] Beck, AT.: Depression: Causes and Treatment, University of Pennsylvania Press, Philadelphia, 1967.

[13] Montgomery, SA. \& Asberg, M.: A new depression scale designed to be sensitive to change, British Journal of Psychiatry, 134, 1979.

[14] Davidson, J. Turnbull, CD. Strickland, R. et al.: The Montgomery-Asberg Depressive Scale: Reliability and Validity, Acta Psychiatric Scandinavica, 74, 1986.

[15] Goldman, RS. Robinson, D. Grube, BS. Hans, RA. et al.: General Psychiatric Symptoms Measures, In Rush, AJ. et al, (eds.).: Handbook of Psychiatric Measures, American Psychiatric Association, Washington DC, 2000.

[16] Holmes, A. Christells, N. Arnold, C.: Depression and chronic pain, Medical Journal of Australia, 1, 2012.

[17] Bair, MJ. Robinson, RL. Katona, W. Kroenke, K.: Depression and pain comorbidity: A literature review, Archive of Internal Medicine, 163, 2003.
[18] Larson, SL. Clark, MR. Eaton, WW.: Depressive disorders as a long-term antecedent risk factor for incident back pain: A thirteen-year follow-up study from the Baltimore Epidemiological Catchment Area sample, Psychological Medicine, 34, 2004.

[19] Bodner, DR. Seftel, AD. Ducharme, SH.: Sexual and Psychological Aspects of Rehabilitation After Spinal Cord Injury, In Stoudemire, A. Fogel, SB. Greenberg, DB. (eds.): Psychiatric Care of the Medical Patients, $2^{\text {nd }} \mathrm{ed}$, Oxford University Press, New York, 2000.

[20] Zepinic, V. \& Kuzmanovski, B.: Support person (Co-therapist) in the Treatment of Panic Disorder, Canadian Social Science Journal, 12, 2017.

[21] Campbell, P. Hope, K. \& Dunn, KM.: The Pain, depression, disability pathway in those with low back pain: a moderate analysis of health locus of control, Journal of Pain Research, 10, 2017.

[22] Srivastava, S. Yadav, P. Panchal, BN. et al.: Association of depression and chronic lower-back pain, Archives of Psychiatry and Psychotherapy, 4, 2018. 